\title{
HLA Class I Histocompatibility Antigen, CW- 1 Alpha Chain Protein
}

National Cancer Institute

\section{Source}

National Cancer Institute. HLA Class I Histocompatibility Antigen, Cw-1 Alpha Chain

Protein. NCI Thesaurus. Code C62760.

HLA class I histocompatibility antigen, Cw-1 alpha chain protein (366 aa, $41 \mathrm{kDa}$ ) is encoded by the human HLA-C gene. This protein plays a role in the presentation of foreign antigens to the immune system. This class of antigens is associated with certain disease manifestations such as spondylarthritis, psoriasis and multiple myeloma. 